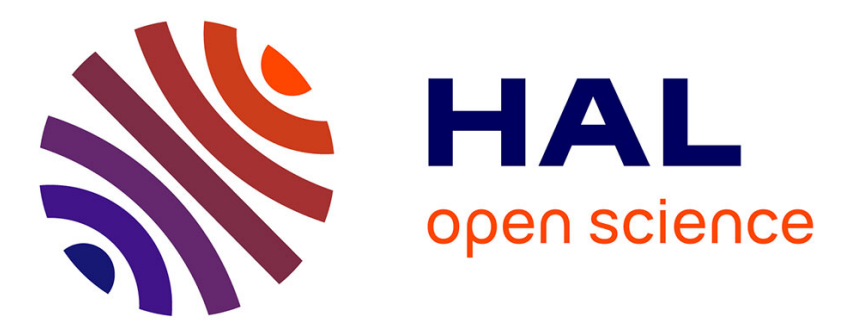

\title{
L'acquisition des préférences alimentaires : le cas du goût sucré
}

Sophie Nicklaus

\section{To cite this version:}

Sophie Nicklaus. L'acquisition des préférences alimentaires: le cas du goût sucré. Le goût du sucre. Plaisir et Consommation, Editions Autrement, pp.168, 2010, Collection Mutations, 978-2746714403. hal-01137042v2

\section{HAL Id: hal-01137042 \\ https://hal.science/hal-01137042v2}

Submitted on 2 Aug 2017

HAL is a multi-disciplinary open access archive for the deposit and dissemination of scientific research documents, whether they are published or not. The documents may come from teaching and research institutions in France or abroad, or from public or private research centers.
L'archive ouverte pluridisciplinaire HAL, est destinée au dépôt et à la diffusion de documents scientifiques de niveau recherche, publiés ou non, émanant des établissements d'enseignement et de recherche français ou étrangers, des laboratoires publics ou privés. 
Cet ouvrage a reçu le soutien de I'Institut Benjamin Delessert. Cet institut a pour mission de financer des projets de recherche universitaires et de contribuer à la diffusion des connaissances. II est soutenu par la filière betterave-canne-sucre française.

Le suivi éditorial de cet ouvrage a été assuré par Anne-Charlotte Sangam.

Graphiques : Jules Grandin.

(c) 2010, Éditions Autrement, 77 rue du Faubourg-Saint-Antoine, 75011 Paris. Tél. : 01447380 00. Fax : 01447300 12. E-mail : contact@autrement.com 


\section{INTRODUCTION}

Marie-Sylvie Billaux

En morceaux ou en poudre, blanc ou roux, le sucre est un ingrédient dont nous apprécions la saveur dès les premières heures de notre vie. Il est synonyme de dessert, de plaisir, de gourmandise, d'enfance...

Mais le sucre est doté de nombreuses facettes qui rendent son approche plus complexe qu'il n'y paraît au premier abord. Voilà pourquoi le choix d'une approche multidisciplinaire s'est tout de suite imposé lorsque nous avons décidé de consacrer un ouvrage au sucre et au sucré. Nous avons donc convié des spécialistes d'horizons variés pour guider le lecteur chapitre après chapitre dans l'univers du sucre. Seront ainsi successivement abordés le plaisir et le goût que nous avons pour le sucre, l'histoire de sa production et de sa consommation, les discours véhiculés à son sujet par la société et les médias ainsi que la relation conflictuelle qu'il entretient avec le consommateur, entre désir et interdit.

Si un parcours gourmand entre mets et mots inaugure l'ouvrage, la mise en perspective historique constitue une approche essentielle permettant d'expliquer les raisons d'un glissement de perception au fil du temps, le passage d'une idéalisation du sucre à un ostracisme sensible. Cela permet ainsi de comprendre les niveaux actuels de consommation du sucre et des produits sucrés en France. Car l'interdit règne toujours. Le débat opposant les tenants de la morale aux doux gourmands perdure, même si le discours culturel et social encadrant la consommation du sucre tend à s'éloigner au profit d'une gestion désormais individuelle de la consommation.
Certes, le sucre dote les aliments d'une saveur agréable aux papilles mais, surtout, il détient des propriétés indispensables à la texture, la coloration, la conservation, etc., mises à profit quotidiennement par celui qui cuisine, le pâtissier ou l'industriel. Aussi est-il utile de se familiariser avec ces propriétés pour comprendre les difficultés et les enjeux qu'il y a à modifier une recette en limitant cet ingrédient pour le remplacer par un autre.

Il était par ailleurs inenvisageable de parler du sucre sans aborder le goût sucré et son évolution au cours de la vie, l'acquisition des préférences alimentaires, le plaisir qui les guide et conditionne nos comportements vis-à-vis de la nourriture. Pour débattre des injonctions sur de prétendues ou réelles consommations excessives de sucre, il était important de s'intéresser aux niveaux de consommation des Français, l'objectif étant également de mieux cernẻr la spécificité de notre modèle alimentaire qui place le plaisir et la convivialité au premier plan, à l'inverse des Américains qui pensent prioritairement en termes de nutrition.

L'addiction, si couramment évoquée par le grand public, est également au cœur de notre ouvrage. Cette question est à manier avec circonspection et il y a lieu de distinguer ce qui relève du domaine de la croyance de la réponse scientifique à une question complexe. De même, nous tenterons de déjouer les a priori et les idées reçues affectant le domaine du conseil diététique et des régimes, notamment en ce qui concerne les prescriptions délivrées aux patients souffrant de surcharge pondérale ou de diabète.

Enfin, les chemins de la création pâtissière se devaient d'être parcourus. La présentation du travail de l'artiste qui imagine et construit un univers de goût, de sensations et de plaisir sera le point d'orgue de cet ouvrage. 


\section{LE GOÛT DU SUCRE \\ Plaisir et consommation}

Le goût pour le sucre nous rappelle, dit-on, la douce saveur du lait maternel. Saveur que l'on déguste avec délices sous multiples formes. En morceaux ou en poudre, blanc ou roux, candy ou cristallisé, le sucre est synonyme de dessert, de plaisir, de gourmandise, d'enfance... Chaque jour, il est utilisé à la maison ou par le pâtissier et l'industriel en raison de ses propriétés indispensables à la te̊xture, la coloration et la conservation des mets.

Or sa consommation est malmenée par le discours social et les médias, et le gourmand se sent tiraillé entre désir et interdit. Pourtant, il fut un temps où le sucre était un aliment idéalisé. L'histoire de sa production et de sa consommation est à la mesure des débats qu'il suscite aujourd'hui.

Outre la mise en perspective historique, ce livre revient avec vigilance sur la question de l'addiction. Médecins, nutritionnistes et diététiciens n'hésitent pas à remettre en cause les idées reçues dans le domaine de la diététique et notamment les prescriptions délivrées aux patients souffrant de surcharge pondérale ou de diabète. Si alimentation rime avec nutrition, le plaisir ne doit toutefois pas être oublié ! Pierre Hermé nous fera ainsi découvrir les clés du travail de l'artiste imaginant et construisant un univers de nouvelles alliances et de saveurs.

C'est donc à un délicieux voyage dans l'univers du sucre et du sucré que nous invite ce livre.

Dirigé par Marie-Sylvie Billaux.

Avec Gérard Apfeldorfer, Ingrid Astier, Dominique-Adèle Cassuto, Franck Cochoy, Julia Csergo, Claude Fischler, Gisèle Harrus-Révidi, Pascale Hébel, Pierre Hermé, André Holley, Serge Michels, Sophie Nicklaus, Philippe Reiser, Gérard Slama. 
L'ACQUISITION DES PRÉFÉRENCES ALIMENTAIRES :

LE CAS DU GOÛT SUCRÉ

Sophie Nicklaus

Le goût sucré est apprécié dans toutes les cultures et par plusieurs espèces animales (rongeurs, primates...), à l'exception notable des chats qu ne possèdent pas le récepteur permettant d'identifier ce goût ${ }^{1}$ L'homme ne fait pas exception à cette règle et apprécie fortement cette saveur. L'attraction pour des aliments très sucrés semble néanmoins caractéristique de l'enfance. Différentes observations soulignent, en effet, l'attrait particulier du nouveau-né ou du nourrisson pour le goût sucré ; attrait qui évolue au cours du développement. Différents mécanismes sous-tendent l'acquisition de ces préférences pour le goût sucré, qui varient d'un individu à l'autre.

\section{Le goût sucré de l'enfance}

Comment aborder la question de l'évolution de l'attraction pour le goût sucré au fil d'une vie ? En première approche, la lecture de données glo-

1. Berridge, K. C., " Measuring hedonic impact in animals and infants: microstructure of affective taste reactivity patterns », Neurosci Biobehav Rev, $\mathrm{n}^{\circ} 24,2000$, p. 173-198; Hladik, C. M., Simmen, B., « Taste perception and feeding behavior in nonhuman primates and human populations ", Evol. Anthropol, 1997, p. 58-71; Li, X, Li, W. Wang, Hi et "Pseudogenization of a sweet-receptor gene accounts for cats'indifference toward sugar PLoS Genetic's, $\mathrm{n}^{\circ} 1$, 2005, p. 27-35. bales de consommation alimentaire est utile. Ainsi, l'analyse des consommations alimentaires aux États-Unis entre 1994 et 1996 indique que la part des sucres ajoutés dans l'alimenta 2-5 ans à la période 12-17 ans accroît de la période des âges avancés. femmes ${ }^{2}$. arance, la contribution des glucides simples à l'ensemble des apports énergétiques diminue de la période 2 6 ans (autour de $23 \%$ ) à l'âge adulte (autour de $15 \%$ ).

L'étude des préférences pour des aliments plus ou moins sucrés reflète aussi cette forte attraction pour des variantes très sucrées pendant l'enfance et l'adolescence : lorsunt perception d'orence : lorsque les participants doivent évaluer leur 12 ans préfèrent leses contenant des ajouts de sucre, les enfants de 6 à sucrées que les les orangeades les plucrées et les peivent moins sucrées que les adultes ${ }^{3}$. L'attirance pour des aliments très sucrés et la consommation importante de ce type d'aliments semblent être, de manière non exclusive, caractéristiques de l'enfance et de 'raclescence.

À la différence des autes saveurs (salé, aciet de l'adolescence. le sucré est appréce des autres saveurs (salée, acide, amère et umami) le sucré est apprécié dès la naissance. Le nouveau-né manifeste cet engouement par des expressions traduisant la satisfaction ${ }^{4}$. Cette appréciation précoce se manifeste avant même que le nouveau- Cé eu la possibilité de consomme nouveaú ai '́appren un aliment sucré et donc l'opportunité d'apprendre l'association entre son goût et son apport énergétique. Les substances de saveur acide, amère ou salée déclenchent généralement chez le nouveau-né des réactions qui semblent traduire un affect négatif : grimaces, pleurs, salivation...

Le nouveau-né est un fin connaisseur du goût sucré. Ainsi, lorsqu'on lui présente une stimulation sucrée, son visage se relaxe, il se lèche les lèvres, manifeste des mouve relaxe, il sourit même parfois ${ }^{5}$. Ces

2. Guthrie, J. F., Morton, J. F., "Food sources of added sweeteners in the diets of Americans ", J. Am. Diet. Assoc., n 100, 2000, p. 43-51.

3. Zandstra, E. H., Graaf, C. de, « Sensory perception and pleasantness of orange beverages from childhood to old age ", Food Qual. Pref., n ${ }^{\circ}$ 9, 1998, p. 5-12.

4. Steiner, J. E., "Human facial expressions in response to taste and smell stimulation", Adv. Child Dev. Behav. n 13, 1979, p. 257-295 ; Rosenstein facial responses to four basic tastes in newborns ", Child Dev, ${ }^{\circ} 59$, 1988, p. 1555-1568. 5. Ganchrow, J. R., Mennella, J. A., « The ontogeny of huma ne, 1988, p. 1555-1568. R. L., Dekker, M., Handbook of Olfaction and Gustation, Nety 
manifestations de plaisir par des observateurs ne connaissant pas la nature de la stimulation ${ }^{6}$. Une stimulation sucrée est également associée à un allongement des cycles de succion (pour mieux «savourer » ?) et à une accélération du rythme cardiaque, proportionnelle à la concentration de sucre ${ }^{7}$. Différentes approches attestent de la subtilité du nouveau-né à distinguer des goûts sucrés variables : en effet, i discrimine leurs concentrations e̊t préfère les solutions plus sucrées jusqu'à un optimum au-delà duquel sa préférence diminue ${ }^{9}$. Enfin, le nouveau-né différencie des sucres aux pouvoirs sucrants inégaux (saccharose, glucose, fructose, lactose) lorsqu'ils sont proposés aux mêmes concentrations : il préfère les sucres dont le pouvoir sucran est le plus élevé (il préfère le saccharose au glucose et au lactose ; et le fructose au glucose)

Certaines pratiques pédiatriques s'appuient sur l'appréciation de la saveur sucrée par le nouveau-né pour réduire la douleur lors d'interventions bénignes ${ }^{10}$. Ainsi, lorsqu'un nouveau-né subit une piqûre, la présentation d'une solution de saccharose diminue généralement l'intensité des indicateurs de la douleur, tant physiologiques (fréquence cardiaque) que comportementaux (latence et durée des pleurs,

\section{Rosenstein, D., et Oster, H., art. cit.}

7. Crook, C. K., "Taste perception in the newborn infant ", Inf. Behav. Dev, n ${ }^{\circ} 1,1978$ p. 52-69 ; Ashmead, D. H., Reilly, B. M., Lipsitt, L. P., « Neonates'heart rate, sucking rhythm, and sucking amplitude as a function of the sweet taste ", J. Exp. Child Psychol. $n^{\circ} 29,1980$, p. 264-281 ; Crook, C. K., Lipsitt, L. P., « Neonatal nutritive sucking: effects of taste stimulation upon sucking rhythm and heart rate ", Child Dev., ${ }^{\circ} 47$, 1976, p. 518-522, Daniel, P. A. Rockwood Zakreski, J, Lipsitt, L. P., “ Iffects of aud to and taste stimulation on sucking heart rate, and movement in the newborn ", In Behav. Dev., nº 5, 1982, p. 237-247 ; Porges, S. W., Lipsitt, L. P., « Neonatal responsivity to gustatory stimulation: the gustatory-vagal hypothesis ", Inf. Behav. Dev., n ${ }^{\circ} 16,1993$ p. 487-494.

8. Desor, J. A., Maller, O., Turner, R. E., « Preference for sweet in humans. Infants, children and adults Ganchrow, J. R., Steiner, J. E., Daher, M., « Neonatal facial expressions in response to different qualities and intensities of gustatory stimuli ", Inf. Behav. Dev., nº 6,1983 p. $473-484$

9. Desor, J. A., Maller, O., Turner, R.E., « Taste in acceptance of sugars by human infants ", J. Comp. Physiol. Psychol., n ${ }^{\circ} 84,1973$, p. 496-501.

10. Stevens, B., Yamada, J., Ohlsson, A., « Sucrose for analgesia in newborn infants undergoing painful procedures ", Cochrane Database Syst Rev, 2004, CD001069. mimiques faciales ${ }^{11}$ ). L'effet antidouleur est optimal si la solution de sucre est administrée deux minutes avant la piqûre ; et il est renforcé par la succion d'une tétine ${ }^{12}$. La concentration de saccharose nécessaire pour produire un tel effet est plus importante chez l'enfant prématuré (24\%) que chez l'enfant né à terme (12\%). De plus, l'application de sucre répétée trois jours de suite produit toujours l'effet antidouleur : l'organisme ne s'y adapte pas ${ }^{13}$. Cet effet antidouleur du sucre est spécifiquement lié au goût sucré et non à l'apport énergétique du sucre : l'injection intragastrique de saccharose ne produit pas cet effet antidouleur, alors qu'une solution d'aspartame produit le même effet qu'une solution de saccharose ${ }^{14}$. Cependant, l'effet calmant du sucre est modéré puisqu'il ne permet pas d'atténuer les douleurs liées à des interventions plus douloureuses telles que la circoncision.

\section{Le goût pour le sucre évolue-t-il avec l'âge?}

L'effet antidouleur du goût sucré évolue dans les premiers mois de la vie. Chez le rat, il semble limité à la période antérieure au sevrage et ne se manifeste plus chez l'adulte ${ }^{15}$. Chez l'enfant humain, l'effet aná sique persisterait entre 6 et 9 semaines avec une solution à $12 \%$, mais n'est plus observé à 12 semaines ${ }^{16}$. Une solution plus concentrée

11. Stevens, Yamada, Ohlsson, 2004, ibid.; Blass, E. M., Watt, L. B., "Suckling- and sucrose-induced analgesia in human newborns ", Pain, n 83, 1999, p. 611-623; Abad, F, Diaz, N. M., Domenech, E., Robayna, M., Rico, J., « Oral sweet solution reduces ; Abad, F. behaviour in preterm infants ", Acta Paediatr., n ${ }^{\circ} 85,1996$, p. 854-858.

12. Blass et Watt, ibid ; Akman, I., Ozek, E., Bilgen, H., Ozdogan, T., Cebeci, D., « Sweet solutions and pacifiers for pain relief in newborn infants ", J. Pain., n ${ }^{\circ} 3$, 2002, p. 199-202. 13. Eriksson, M., Finnstrom, O., "Can daily repeated doses of orally administered glucose induce tolerance when given for neonatal pain relief? ", Acta Paediatr., n ${ }^{\circ}$ 93, 2004, p. 246-249. 4. Ramenghi, L. A., Evans, D. J., Levene, M. I., " "Sucrose analgesia": absorptive $246-249$. nism or taste perception? ", Arch. Dis. Child Fetal Neonatal Ed., n 80 , 1999, p. 146-147 Barr, R. M., Pantel, M. S., Young, S. N., Wright J H w The response of cring n. 146-147; sucrose: is it a "sweetness" effect? ", Physiol. Behav, n० 66,1999, p. 409.417. newborns to 15. Anseloni, V. C. Z., Weng H? P. Pe

cited by intralgesia elicited by intraoral sucrose in acute and persistent pain models ", Pain, $\mathrm{n}^{\circ} 97,2002$, p. 93-

16. Blass, E. M., Camp, C. A., "Changing determinants of crying termination in 6 - to 12 week-old human infants ", Dev. Psychobiol., $\mathrm{n}^{\circ} 42$, 2003, p. 312-316. 
( $24 \%$ ) permet d'observer un effet analgésique chez des nourrissons de 2 et 4 mois $^{17}$ et également chez des enfants de 5 à 11 ans. Ceux qui apprécient plus le goût sucré ressentent plus fortement son effet antidouleur ${ }^{18}$.

La préférence pour le goût sucré, prononcée à la naissance, devient plus modérée au cours de la petite enfance ${ }^{19}$. Pendant les deux premières années, la réponse à une stimulation sucrée varie de la préférence marquée à l'indifférence. En moyenne, les solutions plus concentrées sont préférées. Une solution de lactose est appréciée à 3, 6 mois et 12 mois, mais à partir de 6 mois, elle est autant goûtée qu'une solution de $\operatorname{sel}^{20}$.

Malgré la relative diminution de la préférence pour la saveur sucrée entre la naissance et la petite enfance, cette saveur reste néanmoins appréciée dans l'enfance. Une étude menée chez des préadolescents ( 9 à 15 ans) montre qu'ils se tournent plus vers les solutions très sucrées que les adultes ${ }^{21}$, comme le souligne l'exemple de l'orangeade. Cette préférence semble associée à la période de la préadolescence : les mêmes participants, dix ans plus tard, préfèrent des solutions moins sucrées ${ }^{22}$. L'attraction des enfants et des adolescents pour les aliments

17. Hatfield, L. A., Gusic, M. E., Dyer, A.-M., Polomano, R. C., « Analgesic properties of oral sucrose during routine immunizations at 2 and 4 months of age ", Pediatrics, $\mathrm{n}^{\circ} 121,2008$, p. 327-334.

18. Miller, A., Barr, R. G., Young, S. N., « The cold pressor test in children: methodologica aspects and the analgesic effect of intraoral sucrose ", Pain, n ${ }^{\circ} 56,1994$, p. 175-183 Pepino, M. Y., Mennella, J. A., « Sucrose-induced analgesia is related to sweet preferences in children but not adults", Pain, n 119,2005 , p. 210-218.

19. Beauchamp, G.K., Cowart, B. J., "Development of sweet taste ", in Dobbing, J., Sweetness, Springer-Verlag, Berlin, 1987, p. 127-140 ; Beauchamp, G. K., Moran, M., "Acceptance of sweet and salty tastes in 2-year-old children", Appetite, n 5, 1984, p. 291 305 ; Vasquez, M., Pearson, P.B., Beauchamp, G.K., « Flavor preferences in malnurished mexican infants ", Physiol. Behav., n 28, 1982, p. 513-519.

20. Schwartz, C., Issanchou, S., Nicklaus, S., « Developmental changes in the acceptance of the five basic tastes in the first year of life ", Br. J. Nutr., $\mathrm{n}^{\circ} 102,2009$, p. 1375-1385.

21. Zandstra et de Graaf, ibid. ; Desor, J. A., Greene, L. S., Maller, O., « Preference for sweet and salty in 9 - to 15 -year-old and adult humans ", Science, $n^{\circ} 190,1975$, p. 686-687. 22. Desor, J. A., Beauchamp, G. K., "Longitudinal changes in sweet preferences in humans ", Physiol. Behav., n 39, 1987, p. 639-641 ; Pepino, M. Y., Mennella, J. A., "Children's liking of sweet tastes and its biological basis" in Spillane, W. J. Optimising 65. Sucrés pourrait s'expliquer par leurs besoins énergétiques relativement
élevés $^{23}$.

Selon une étude s'intéressant à l'enfance jusqu'au début de l'âge adulte $^{24}$, les préférences alimentaires se canalisent depuis la petite enfance. Ainsi, les enfants mangeant plus de légumes au cours petite petite enfance continuent à en consommer ultérieurement. rence pour les aliments (très) sucrés naissant literieurement. La préfése maintenir à l'âge adulte. Cependant, très peurs de l'enfance pourrait ce sujet. Seule une étude amendant, très peu d'études ont porté sur montre une diminution relativement ancienne (1987), lescence à l'âge adulten relative des préférences pour le sucre de l'adode la saveur sucrée sont pas connues.

Les mécanismes d'acquisition des préférences pour le goût sucré

Le débat entre l'inné et l'acquis des préférences alimentaires semble partiellement résolu. Un consensus se dégage, indiquant que pour une large part, les préférences alimentaires sont forgées par les expériences, donc acquises au cours du développement. Le cas des aliments sucrés est un peu différent, étant donné l'appréciation élevée de la saveur sucrée dès la naissance. Elle peut néanmoins être augmentée sous l'effet de plusieurs mécanismes.

La répétition de la consommation peut renforcer l'appréciation spontanée. La préférence pour l'eau sucrée manifestée dès la naissance est maintenue chez le nourrisson ayant reçu de l'eau sucrée pendan

in Murphy, in Muph, C., Cain, W. S., et Hegsted, D. M., Nutrition and the Chemical Senses it life Recent Advances and Current Research Needs, New York, The New York Acas senses in Agin. 1989, p. 243-250 ; Graaf, C. de, Zandstra, E. H. "Sweetness intensity ademy of Sciences, children, adolescents, and adults ", Physiol. Behav, no $67,199{ }^{\circ}, \mathrm{p}$. $513-520$ and pleasantness in 24. Nicklaus, S., Boggio, $V$, Chabanet, C., ISs., 1067,1999 , p. 513-520. variety seeking in childhoo, ado p. 289-297 ; Nike in early adult life", Appetite, ${ }^{\circ} 44,2005$ p. $289-297$, Ncklaus, S., Boggio, V., Chabanet, C., Issanchou, S., «A prospective study food preferences in childhood ", Food Qual. Pref., n ${ }^{\circ} 15,2004$, p. $805-818$. 25. Desor et al., ibid. 
les six premiers mois, alors qu'elle diminue chez les autres. Cet effet est encore apparent à 2 ans $^{26}$. Il n'est pas généralisable à d'autres boissons : tous les enfants de 2 ans, exposés ou non à de l'eau sucrée, préfèrent un jus de fruits sucré au même jus non sucré. En revanche, une exposition pendant trois mois à des " petits pots » moins sucrés que les versions habituelles n'a pas modifié l'attirance pour le sucré d'enfants de 7 mois $^{27}$. Enfin, les enfants de 4 à 7 ans à l'alimentation desquels les mères ajoutent régulièrement du sucre préfèrent les jus de pomme et les céréales plus sucrées ${ }^{28}$.

La familiarité croissante avec l'aliment pourrait expliquer les apprentissages liés aux expositions répétées. Des enfants de 4 à 5 ans ayant goûté quinze fois du tofu salé, sucré ou nature, préfèrent, quelques semaines plus tard, la version à laquelle ils ont été exposés ${ }^{29}$. Dans ce cas, l'exposition à un aliment n'agit pas sur l'attrait pour les différentes déclinaisons de celui-ci.

Au-delà de l'exposition répétée à un aliment, son goût facilite l'acquisition de la préférence. Ainsi, après huit jours d'exposition, les enfants de 6 à 11 ans préfèrent une orangeade sucrée, mais pas une orangeade dont la saveur est nettement plus acide ${ }^{30}$.

L'appréciation des aliments sucrés est renforcée par leur contenu énergétique. Le corps humain est conçu pour associer une préférence élevée à un apport énergétique important, comme dans le cas des aliments gras et/ou sucrés. L'apport énergétique est un puissant moteur de nos goûts alimentaires expliquant en partie le rejet des légumes, souvent pauvres en énergie, et les succès aléatoires des produits « allégés ».

Cet effet de l'exposition aux aliments sucrés s'observe à l'échelle des groupes culturels. Ainsi, une étude nord-américaine montre que la préférence d'enfants afro-américains pour des solutions sucrées est

26. Beauchamp et Moran, art. cit.

27. Brown, M. S., Grunfeld, C. C., « Taste preferences of infants for sweetened or unsweetened foods ", Res. Nurs. Health, $\mathrm{n}^{\circ} 3,1980$, p. 11-17.

28. Liem, D. G., Mennella, J. A., "Sweet and sour preferences during childhood: role of early experiences ", Dev. Psychobiol., n 41,2002 , p. 388-395.

29. Sullivan, S. A., Birch, L. L., «Pass the sugar, pass the salt : experience dictates preference ", Dev. Psychol., n 26, 1990, p. 546-551.

30. Liem, D. G., Graaf, C. de, « Sweet and sour preferences in young children and adults: role of repeated exposure", Physiol. Behav., n 83, 2004, p. 421-429. bien supérieure à celle d'enfants caucasiens ${ }^{31}$. Dans ce cas, on ne peut exclure l'impact de différences génétiques entre les deux populations : néanmoins, la plus grande fréquence de consommation d'aliments caucasienne peut être à afro-américaine par rapport à la population le la différence observée.

parents. Ils peuvent intervenir de essentiellement transmises par le de l'attraction de leur contrôle parental influence le sur la consommation d'aliments sucrés restreignent la confents dont les parents pour des concentrationmation de sucre expriment des préférences libres de leur consommation sucre plus élevées que les enfants laissés ble effectif à consommation ${ }^{32}$. Ainsi, le contrôle parental strict seml'attraction pour lerme, mais il ne fait généralement que renforcer sucrés) sont parfois utients très appréciés (souvent familiale, ou comme parents pour fêter une occasion pense en échange d'un comportement pour ces aliments légumes...). L'attraction initiale étroite pour unents n'en est que renforcée ${ }^{33}$. Ainsi, la voie semble sucre.

lependant, l'attraction des enfants pour le sucre peut présenter quer le goût amer intérêt pratique puisque le sucre permet de masfruits. Un légume certains légumes ou le goût acide de certain blement exposé est plus apprécié d'enfants de 5 ans s'il a été préalajus de pamplemousse (chez des sucrée ${ }^{34}$. Il en va de même avec du (chez des jeunes adultes de 19 an 5 ans) et du brocol

31. Pepino, M. $Y$, Ment

ucrose preference ", Chem. Senses, " ${ }^{\circ} 30$. p. I319-I320

and 5-year-old children: role of parents, "Sweet preferences and sugar consumption of 4 33. Birch, L. L., Zimmerman, S. I, Hind, Appetite n ${ }^{\circ} 43,2004$, p. 235-245.

the formation of children's food preferences " The influence of social-affective context on 34. Havermans, R. C., Jansen, A. "Inces ", Child Dev., n 51, 1980, p. 856-861.

vour-flavour learning ", Appetite, " Increasing children's liking of vegetables through fla8, 2007, p. 259-262.

adults ", Appetite, $\mathrm{n}^{\circ} 50,2008, \mathrm{p}$. 
lorsque l'aliment est offert sans sucre ajouté. Ainsi, l'ajout de sucre à des légumes amers permet d'en augmenter l'appréciation par les enfants.

\section{Des appréciations variables du goût sucré}

L'appréciation du goût sucré est ancrée dans l'enfance. Cependant, elle varie d'un enfant à l'autre, tout comme elle varie d'un adulte $l^{\prime} a u t r e^{36}$. Les variations de préférences traduisent une différence de détection de l'intensité de la saveur sucrée ou une réactivité affective variée ${ }^{37}$. Les progrès récents de la biologie moléculaire permettent de comprendre le mécanisme de détection du sucre. Dans l'état actuel des connaissances, il semble que toutes les molécules sucrées (naturelles ou de synthèse) soient détectées par une association de deux récepteurs gustatifs, T1R2 et T1R $3^{38}$, alors que les molécules amères, très nombreuses et de nature chimique très variée, sont détectées par vingt-cinq récepteurs différents.

Des variations génétiques d'un des deux récepteurs pourraient conduire à des perceptions variables d'un individu à l'autre. De telles variations génétiques ont largement été étudiées chez les rongeurs. Elles sont importantes et se traduisent chez la souris par des attraction très différentes pour la saveur sucrée d'une souche de souris à une autre $^{39}$. Chez l'homme, on a identifié plus de variations génétiques pour le récepteur T1R2 que pour le récepteur T1R $3^{40}$, mais leurs consé-

36. Duffy, V., Hayes, J. E., Dinehart, M. E., « Genetic differences in sweet taste perception ", in Spillane, W. J., Optimising Sweet Taste in Foods, Cambridge, England, Woodhead Publishing Limited, 2006, p. 30-53.

37. Lony, H., Weingarten, H. P., « Facial expressions and genetic sensitivity to 6-normalpropylthiouracil predict hedonic response to sweet ", Physiol. Behav., nº 52, 1992, p. 75-82. 38. Lindemann, B., " Receptors and transduction in taste ", Nature, $n^{\circ} 413,2001$, p. 219 225 ; Chandrashekar, J., Hoon, M. A., Ryba, N. J. P., Zuker, C. S., « The receptors and cells for mammalian taste ", Nature, $\mathrm{n}^{\circ} 444,2006$, p. 288-294 ; Huang, A. L., Chen, X., Hoon, M. A., et al., "The cells and logic for mammalian, sour taste detection ", Nature, $\mathrm{n}^{\circ} 442$, 2006, p. 934-938.

39. Boughter, J. D. Jr., Bachmanov, A. A., « Behavioral genetics and taste ", BMC Neurosci., $n^{\circ} 8,2007$, Suppl. 3, S3

40. Kim, U. K., Wooding, S., Riaz, N., Jorde, L. B., Drayna, D., « Variation in the human TAS1R taste receptor genes ", Chem. Senses, n ${ }^{\circ} 31,2006$, p. 599-611. quences sur les variations de détection de la saveur sucrée sont encore mal connues et certainement moins importantes que chez la souris. Dans quelques années, la compréhension de la génétique de la détection et de l'appréciation du goût sucré aura progressé.

Des découvertes récentes indiquent que la plupart des récepteurs gustatifs, présents sur la langue, sont également présents à la lumière de certaines cellules intestinales et pourraient contribuer à réguler l'absorption intestinale des nutriments ${ }^{41}$. De nouvelles perspectives s'ouvrent sur la compréhension de l'acquisition des préférences pour le goût sucré.

\section{Le goût sucré, demain}

Pendant des millénaires, l'homme a évolué dans un environnement où les sources de sucre étaient rares. Depuis à peine plus d'un siècle, son appétit naturel pour les aliments sucrés a été de plus en plus satisfat grâce à la culture de la cannen en satisfait a canne et de la betterave à sucre. L'attraction a conduit à l'élaboration d'édulcorants au puissant goût sucré mais moins énergétiques que les sucres naturels ${ }^{42}$. L'impact de la présence de ces ingrédients dans de nombreux aliments ou boissons sur un renforcement éventuel de l'appréciation du goût suré noissons analysé à ce jour. populations un autre domaine, il a été observé dans les ${ }^{43}$ tions plus fortement consommatrices d'aliments riches en amidon ${ }^{43}$ une évolution génétique favorisant la digestion de l'amidon. La présence accrue du goût sucré dans notre alimentation pourrait également favoriser une évolution génétique. L'avenir est riche d'observaions concernant l'attraction pour le goût sucré.

41. Bezencon, C., Le Coutre, J., Damak, S., « Taste-signaling proteins are coexpressed in solitary intestinal epithelial cells", Chem. Senses, ${ }^{\circ} 32$, 2007, p. 41-49 . Maroolskeed Dyer, J., Kokrashvili, Z., et al., «T1R3 and gustducin in gut sense sugars to regolskee, R. F., sion of Na+- glucose cotransporter 1 ", Proc. Natl. Acad. Sci. U. S. A n ${ }^{\circ} 104$, 2007 , $15075-$ 15080 .

42. Cox, T. M., "The genetic consequences of our sweet tooth ", Nat. Rev. Genet., n ${ }^{\circ} 3$,

43. Perry, G. H., Dominy, N. J., Claw, K. G., et al., « Diet and the evolution of human amylase gene copy number variation », Nat. Genet., ${ }^{\circ} 39,2007$, p. 1256-1260. 